\section{Atención de salud de personas transgéneros para médicos no especialistas en Chile}

\author{
ANTONIO ZAPATA PIZARRO ${ }^{1,3}$, KARINA DÍAZ DÍAZ $^{1, a}$, \\ LUIS BARRA AHUMADA ${ }^{2}$, LORENA MAUREIRA SALES ${ }^{4, b}$, \\ JEANETTE LINARES MORENO ${ }^{1}$, FRANCO ZAPATA PIZARRO $^{4}$
}

\section{Healthcare of transgenders by non-specialists in Chile}

The health care demand for transgenders has increased in Chile and worldwide. However, in Chile health care professionals are not trained to understand and face this problem. We herein review issues that should be considered in the training of non-specialist physicians to provide health care to transgenders, issues about terminology of body reassignment treatments and gender identity and the way Chilean professionals should deal with transgender persons.

(Rev Med Chile 2019; 147: 65-72)

Key words: Gender Identity; General Practice; Health Services for Transgender Persons; Sexual and Gender Minorities; Transgender Persons.

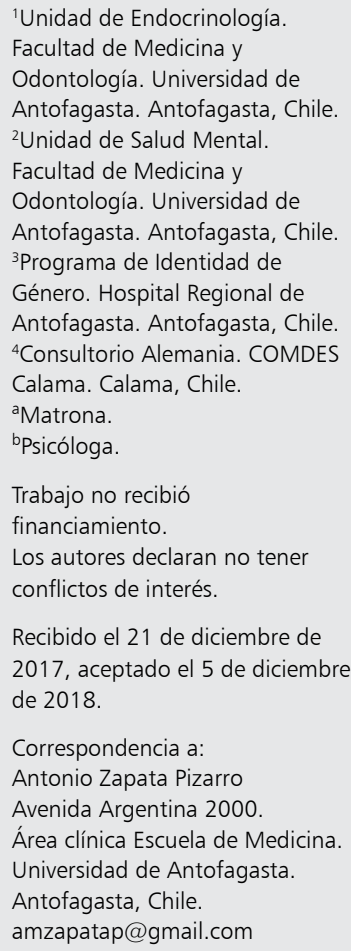

Correspondencia a:

Antonio Zapata Pizarro

Avenida Argentina 2000

Área clínica Escuela de Medicina.

Universidad de Antofagasta.

Antofagasta, Chile.

amzapatap@gmail.com

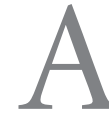

ctualmente es más frecuente la demanda de atención sanitaria de personas transgéneros, por lo que es necesario que el médico, aunque no sea especialista, conozca aspectos generales respecto a la terminología, necesidades de salud, trato médico legal y consideraciones respecto a eventuales efectos de los tratamientos médicos a los que se ven sometidos estos pacientes en su tránsito a la identidad de género sentida.

Para analizar este tema, es necesario definir los términos: sexo, género, determinación sexual, orientación sexual.

El sexo se define según las determinantes biológicas del individuo; en la mayoría de los casos, los recién nacidos son asignados como hombres o mujeres según su sexo anatómico, que para el contexto social, debe buscar ser congruente con una identidad y expresión de género "masculina"(de niño) o "femenina" (de niña). En el caso de que el recién nacido no tenga un sexo anatómico definido, se usa el término intersexual (previamente llamados hermafroditas) ${ }^{1}$.

El género se refiere a la experiencia personal interna de sentirse masculino, femenino o andrógeno, según el contexto cultural (identidad de género). La
Organización Mundial de la Salud (OMS) lo define como "características de hombres y mujeres que están basadas en factores sociales, mientras que el sexo se refiere a las características que vienen determinadas biológicamente"2. Si la persona se identifica con el sexo asignado al nacimiento, se la considera como una persona "cisgénero", si no lo hace, se la considera "transgénero" 3 . La expresión de género que engloba aspectos del comportamiento en los cuales hombres y mujeres son diferentes según la cultura y la etapa histórica (conducta de género masculina o femenina según patrones culturales). En las últimas décadas es más frecuente ver expresiones de conductas de género distintas a las clásicamente conocidas como masculinas o femeninas, incluyendo personas andróginas, bigéneros, o que no se identifican con ninguno de los dos géneros (no binarios) ${ }^{4}$.

La orientación sexual se refiere hacia quien está dirigido el deseo sexual, existiendo personas con orientación homo, hétero, bisexual, pansexual o ninguna (i.e. asexual), dependiendo hacia donde se dirija la atracción erótica-afectiva del individuo, la que no necesariamente está definida por el sexo asignado al nacer ${ }^{1,3}$. 
En suma, tendremos individuos con:

- Sexo cromosómico XX, XY y sus variantes.

- Sexo genital masculino, femenino o intersexuales.

- Orientación sexual homosexual, héterosexual, bisexual, asexual o pansexual.

- Apariencia sexual determinada por el aspecto fenotípico y por la expresión de la identidad o expresión de género masculino, femenino o andrógino, dependiendo del contexto cultural del individuo.

Así, podemos ver que sexo, género y orientación sexual no son sinónimos.

\section{Disforia de género e identidad transgénero}

La disforia de género (DG) es una categoría diagnóstica que describe a las personas transgéneros que sienten un distrés o disforia debido a la discordancia entre su identidad de género y su sexo asignado al nacer, con el que no se identifican ni sienten como propio, esto, en ausencia de un trastorno endocrinológico o cromosómico. Respecto a su clasificación, la DSM-V (Diagnostic and Statistical Manual of Mental Disorders) ya no lo define como trastorno mental, sino la presencia de malestar clínicamente significativo asociado a la condición de género, con una intención de despatologizar esta condición. Asimismo, el CIE-11 (Clasificación Internacional de Enfermedades, OMS), en junio de 2018, quitó la incongruencia de género de la lista de enfermedades mentales y la trasladó al grupo de afecciones de la salud sexual, con el fin de cubrir las importantes necesidades de atención sanitaria de esta población, pero clarificando que no es un trastorno mental ${ }^{5,6}$. Hasta hace unos años, ciertas definiciones, para la población e incluso para los profesionales de la salud, eran sinónimos, sin realmente serlos (transformista, travestie, transgénero, transexual y homosexual)(Tabla 1). Al respecto, movimientos de defensa de los derechos de diversidad sexual y de género han hecho esfuerzos en divulgar, empleando distintos medios, las diferencias de estos términos y visibilizarlos ${ }^{7,8}$.

La identidad transgénero difiere de la transexual en que esta última, además de presentar disconformidad con el sexo y género asignado al nacer, se ha realizado procedimiento de readecuación quirúrgica para su transición de $\operatorname{sexo}^{1,2,7}$. En la presente revisión usaremos, por motivos prácticos, las expresiones personas trans, transgénero o transexual como sinónimos.

\section{Distribución poblacional e índices de salud de las personas transgéneros}

Existe información escasa y poco precisa de la prevalencia de personas transgéneros en la población general; esto porque informes estadísticos poblacionales los excluyen, porque las personas transgéneros se identifican más con denominaciones "hombre" o "mujer" que con la identidad de "transgénero", o bien, por falta de inclusión de la categoría "no binario"(ni masculino ni femenino) en los estudios. Además, en las investigaciones publicadas, habitualmente usan como referencia a las personas transgéneros que solicitan atención de salud de readecuación corporal hormonal o quirúrgica, sin considerar que un alto porcentaje de personas transgéneros se automedican hormonalmente y no asisten a centros de sa$\operatorname{lud}^{9}$, otros estudios tienen problemas metodológicos al definir a la población transgénero ${ }^{10,11}$. En Estados Unidos de Norteamérica, estudios realizados en los últimos años por Blosnich (2013) y Crissman (2017) estiman que $0,5 \%$ de la población se podría definir como transgénero ${ }^{12,13}$. Es muy probable que estos valores subestimen la prevalencia real. Considerando que en Chile no existen datos claros del número de personas trans, podríamos hacer una extrapolación de las cifras norteamericanas a la población chilena del último censo, y estimar que en Chile habrían más de 80.000 personas transgéneros, y cada año existirían cerca de 30 nuevas personas transgéneros que podrían demandar asistencia sanitaria ${ }^{14}$.

Respecto a la morbilidad asociada, el estudio USTS (U.S. Transgender Survey) 2015 en Estados Unidos de Norteamérica ${ }^{15}$ describe que 39\% de la población transgénero sufre grave malestar psicológico (vs $5 \%$ de la población general), que presentan 9 veces más riesgo de intentos suicidas y 5 veces más frecuencia de presentar infección por VIH (mayormente en transgéneros femeninos). El 25\% no buscaba atención médica por temor al maltrato en los servicios asistenciales. En el aspecto social, en el año 2014, un estudio en Estados Unidos de Norteamérica mostró que las personas transgéneros tenían más probabilidad de estar bajo la línea de la pobreza ${ }^{12}$. Por su parte, una revisión sistemática reciente de estudios de violencia motivada por orientación sexual o de género en minorías sexuales señala la presencia de $68 \%$ de violencia física inflingida y $49 \%$ de violencia sexual ${ }^{16}$.

Las bases biológicas que condicionan a las personas transgéneros continúan siendo un misterio, si bien existen estudios que asocian ciertas atipías 
Tabla 1. Definiciones generales en la atención de personas transgéneros

\begin{tabular}{|c|c|}
\hline Término & Definición \\
\hline Género & $\begin{array}{l}\text { Construcción cultural que asigna, en ámbito público y privado, formas de comportamiento } \\
\text { y de roles a las mujeres y los hombres sobre la base de su diferenciación sexual (Definición } \\
\text { de la OMS) }\end{array}$ \\
\hline Identidad de género & Sensación interna del individuo (no visible a otros), de ser hombre o mujer \\
\hline Variantes de género & $\begin{array}{l}\text { Individuo con expresión de género que difiere de las expectativas sociales relacionadas } \\
\text { con el género asignado }\end{array}$ \\
\hline Transgénero & Identidad, expresión y conducta de género difiere del género asignado al nacer \\
\hline Cisgénero & Identidad, expresión y conducta de género concordante con el género asignado al nacer \\
\hline Género no binario & Disconformidad con cualquiera de las dos formas clásicas de género (hombre-mujer) \\
\hline Hombre transgénero & $\begin{array}{l}\text { Persona con características sexuales femeninas, identificada como mujer al nacer, que } \\
\text { ahora se siente hombre }\end{array}$ \\
\hline Mujer transgénero & $\begin{array}{l}\text { Persona con características sexuales masculinas, identificada como hombre al nacer, que } \\
\text { ahora se siente mujer }\end{array}$ \\
\hline $\begin{array}{l}\text { Disforia de género/Desorden } \\
\text { de identidad de género }\end{array}$ & $\begin{array}{l}\text { Término usado en DSM-V y CIE-10 respectivamente, para describir condiciones por las } \\
\text { cuales la no conformidad de género provoca distrés o malestar clínicamente significativo }\end{array}$ \\
\hline Transición & $\begin{array}{l}\text { Tiempo a través del cual la persona comienza a vivir con un género con el que se identifica, } \\
\text { más que con el género asignado al nacer }\end{array}$ \\
\hline Experiencia en vida real & $\begin{array}{l}\text { Adopción por completo del rol de género al cual se adecúa la persona. Es necesario esta } \\
\text { fase antes de los procesos de hormonación y posterior readecuación corporal quirúrgica }\end{array}$ \\
\hline Drag queen & Hombre que se viste de mujer, generalmente con el fin de entretención o performance \\
\hline Drag king & Mujer que se viste de hombre, generalmente con el fin de entretención o performance \\
\hline Transexual & $\begin{array}{l}\text { Usado habitualmente como sinónimo de transgénero. Sin embargo, en algunas publi- } \\
\text { caciones es utilizado para diferenciar a las personas transgénero que ya se sometieron a } \\
\text { procesos de readecuación quirúrgica sexual }\end{array}$ \\
\hline Travestismo & $\begin{array}{l}\text { Incursión temporal en la indumentaria o accesorios del sexo opuesto con el fin de lograr } \\
\text { exitación y placer sexual. Se considera una parafilia }\end{array}$ \\
\hline
\end{tabular}

(Adaptado de referencia 34 y 37 ).

anatómicas o de función cerebral, no se ha logrado demostrar alteraciones en niveles hormonales o de receptores causantes de la presencia de personas transgéneros ${ }^{17,18}$. En las últimas décadas, la prevalencia de las personas transgéneros en todos los rangos etáreos ha ido en aumento ${ }^{19}$. Estudios muestran que un porcentaje importante de los niños y niñas que presentan una discordancia de género en su niñez y adolescencia no la mantienen al llegar a la adultez ${ }^{20}$. Sin embargo, investigadores cuestionan estos resultados, por errores metodológicos y problemas en la definición de niños y adolescentes transgéneros al momento de interrogar a los sujetos, por lo que son necesarios nuevos estudios para poder establecer criterios para definir qué niños y adolescentes mantendrán inconformidad con su sexo biológico hasta la adultez ${ }^{10,11}$.

\section{Salud en las personas transgéneros en Chile}

Estudios respecto a la población transgénero y su atención sanitaria en Chile son escasos. Barrientos y cols. (2010-2014) han realizado investigaciones sobre percepción de bienestar en personas transgéneros en Chile, reportando que estas sufren altos niveles de victimización y discriminación ${ }^{21,22}$. Las personas transfemeninas muestran menores niveles de felicidad, de satisfacción con su vida, mayores niveles de ansiedad, depresión, somatización y hostilidad al compararlas con una muestra de personas homosexuales $^{23}$. En la Primera Encuesta Nacional de Clima Escolar (2016), 52\% de los y las adolescentes trans declararon haber sido acosados/as físicamente y $88 \%$ reportó haber sido insultado/a por sus compañeros/ as por su expresión de género ${ }^{24}$. 
Investigaciones y organizaciones chilenas de diversidad sexual informan que la población trans, en especial femenina, es vinculada a comercio sexual, en parte porque sufren la exclusión social, discriminación y la poca capacidad de incorporación al mundo laboral ${ }^{7,21}$. La segregación social a la que esta población ha sido expuesta favorece la presencia de dobles vidas, de discriminación de personas identificadas como transexuales (transfobia), exclusión o autoexclusión del régimen educacional, lo que acarrea la dificultad de hallar ambientes laborales que los/las acepten ${ }^{7,8}$. Además, en el aspecto de acceso a salud es escasa la oferta de políticas públicas, las que están enfocadas en particular a la prevención de VIH/SIDA, sin acoger las otras demandas de salud de este grupo, lo que frecuentemente lleva a la automedicación (hormonal y aplicación de sustancias de uso industrial no médico con fines estéticos, como silicona u otros) y, en casos extremos, la automutilación ${ }^{7,24}$.

\section{Intervenciones en salud en las personas transgéneros}

Haciendo eco de la descripción de DSM V sobre DG y el intento de despatologizar las identidades trans, es necesario considerar que las personas transgéneros, aun cuando no tengan una patología desde el punto de vista psiquiátrico, tienen necesidades de salud. Para la OMS, salud es "el estado de bienestar físico, psíquico y social; no la mera ausencia de enfermedad" y sobre la salud sexual, añade que "requiere un enfoque positivo y respetuoso de la sexualidad y de las relaciones sexuales, las que deben ser placenteras y seguras, libres de toda coacción, discriminación y violencia"18. Esto es fundamental en el foco no patologizante de la atención de salud de las personas trans. Existe evidencia del beneficio que presenta el acceso a programas de adecuación sexual en su vida afectiva, social y laboral. En una revisión sistemática de pacientes transgéneros sometidos a tratamientos de hormonación y readecuación sexual, se indica que cerca de $80 \%$ logra insertarse a la vida laboral y mejoran su calidad de vida, $78 \%$ mejora sus síntomas psicológicos y $72 \%$ mejora su función sexual $^{26,27}$. En niños o adolescentes transgéneros, el tratamiento de retraso puberal ha mostrado ser seguro, con buena satisfacción para el/la paciente y de carácter reversible, pudiendo considerarse como un tiempo para una exploración de la identidad de género sentida y del deseo de reasignación sexual en el proceso de maduración sicológica ${ }^{28}$.

Es importante aclarar que no todas las personas transgéneros tienen como meta una readecuación quirúrgica sobre su sexualidad. En ocasiones, solo cambios en la expresión de género o bien el proceso de hormonación hacia el género sentido es suficiente para lograr un bienestar psicosocial ${ }^{29}$.

\section{Intervenciones de salud en personas transgéneros por el Ministerio de Salud de Chile (MINSAL) y organizaciones científicas nacionales}

En relación a la apertura sociocultural que ha enfrentado el país en las últimas décadas y el aumento de consultas de personas transgéneros en los centros de salud, el MINSAL ha redactado documentos (circulares) para orientar a los profesionales de la salud y personal administrativo de consultorios y hospitales sobre el trato que deben recibir estos pacientes.

La circular 34 (MINSAL, 2011) menciona que las personas transgéneros deben ser llamadas con el nombre social con el que la persona se identifica, tanto en el trato verbal y atención, así como también en los registros (ficha clínica, brazaletes de identificación, solicitud de exámenes, etc.). En el caso de los registros, deben incluir el nombre legal (consignado en el carnet de identidad) y el nombre social. Para la hospitalización, la circular 34 aclara que la persona debe ser hospitalizada según el aspecto externo que presente en sector de hombres, mujeres o habitaciones individuales, según la propia disponibilidad del establecimiento. Además, menciona que la entrega de información sobre el estado de salud puede ser dada a personas cercanas no familiares (en caso que sea posible $)^{30}$.

La circular 21 (MINSAL, 2012), por su parte, junto con reforzar las recomendaciones de la circular 34, solicita registrar en los antecedentes médicos si el paciente está en proceso de transición hacia otro género o sexo, además de aclarar que en el caso de que la persona transgénero no pida voluntariamente ser identificada según su nombre y género social, el profesional deberá informar sobre la existencia de esta circular y preguntar cómo prefiere ser tratado/ $/ \mathrm{a}^{31}$.

En los últimos años, el MINSAL, siguiendo la guía de organizaciones internacionales respecto al tema, ha tratado de dar lineamientos respecto a la atención de salud de las personas trans en la "Vía Clínica para 
la adecuación corporal en personas con incongruencia entre sexo físico e identidad de género" de $2010^{32}$. Con ella se busca orientar a los y las profesionales de salud respecto al apoyo que se le puede entregar a estos pacientes que buscan transitar de un género o sexo a otro y que presentan problemas de incongruencia que superan un mínimo de afectación, donde las atenciones sanitarias sean justificadas. En esta vía clínica se plantea intervenciones de salud mental, pasando por la experiencia en vida real (adopción por completo del rol de género al cual se adecúa la persona, requisito previo a la hormonación), seguido por la adecuación corporal hormonal y quirúrgica en las personas que lo requieran y cumplan requisitos específicos, señalando que la puerta de entrada al sistema de atención es la atención primaria, aunque solo se refiere al problema en mayores de 18 años.

Devoto y cols. (2016), en la Revista Chilena de Endocrinología, realizan una revisión actualizada, con una guía de manejo del tratamiento hormonal de pacientes transgéneros, abarcando su abordaje desde la niñez, pasando por la adolescencia, hasta la adultez ${ }^{33,34}$.

En las últimas décadas, distintos grupos en hospitales regionales han desarrollado protocolos de manejo médico para estos pacientes (Van Buren, Talcahuano, Antofagasta y otros), con el fin de organizar los procesos de derivación y de adecuación sexual en esta población ${ }^{35}$.

Respecto a las coberturas de las prestaciones de salud en personas transgéneros, algunas son cubiertas, pero otras, como vaginoplastías, no están descritas, lo que genera problemas de reembolso por parte de los seguros de salud públicos (FONASA) y privados (ISAPREs) ${ }^{24}$. Recientemente, el Hospital Sótero del Río publicó su casuística de 28 pacientes en tratamiento por DG en tratamiento hormonal, de los que solo 14\% había logrado gonadectomía más genioplastía ${ }^{36}$.

\section{Consideraciones clínicas para el médico general en la atención de pacientes transgéneros en proceso de readecuación sexual}

En vista a que cada vez más médicos y otros profesionales de la salud tendrán que brindar atención de las demandas de salud de personas transgéneros, es importante que se tengan las siguientes consideraciones:
- Recordar el trato por el nombre social de el/ la paciente y que los registros clínicos contengan el nombre social y legal de la persona. Si el paciente no lo solicita en forma explícita, informar sobre la existencia de las circulares 34 y 21 que garantizan el trato por el nombre social de el/la paciente.

- Se sugiere que las personas transgéneros tengan una evaluación psicológica previa al inicio del tratamiento de readecuación corporal hormonal o quirúrgico, para descartar patologías de salud mental que contraindiquen dichos tratamientos ${ }^{28}$. Sin embargo, es importante tener en cuenta que no es obligatoria la solicitud de certificados psicológicos para que la persona transgénero sea apoyada en su readecuación corporal $^{24}$.

- El/la paciente debe dar su consentimiento informado por escrito previo al inicio de la terapia médica hormonal, quirúrgica o ambas.

- El inicio de la readecuación corporal pasa por el tratamiento hormonal, indicado por un endocrinólogo, que será generalmente de por vida y que, al menos, debe tener una duración de 12 meses previo a cualquier cirugía.

- En el caso que el/la paciente cumpla las etapas previamente descritas podría ser candidato/a a readecuación sexual quirúrgica, si es que lo solicita.

- Es muy importante mantener los programas de prevención general en los/las pacientes que están en el proceso de readecuación corporal o que ya lo han completado. Recordar evaluaciones ginecológicas como mamografías y Papanicolau de tamizaje en personas trans-masculinos y evaluaciones de tamizaje de cáncer prostático y evaluaciones mamarias en pacientes trans-femeninos que lo requieran.

- También es importante explorar la presencia de síntomatología depresiva o ansiosa que requieran mayor apoyo y monitorizar la presencia de indicadores de riesgo suicida (Figura 1).

Respecto al tratamiento hormonal, en las personas transexuales femeninas, habitualmente se utiliza estrógenos por vía oral o transdérmica, espironolactona como antagonista androgénico. En personas transexuales masculinas el proceso de hormonación usa testosterona intramuscular o en gel, y progestágenos en los primeros meses para lograr amenorrea ${ }^{26,27,29}$. 


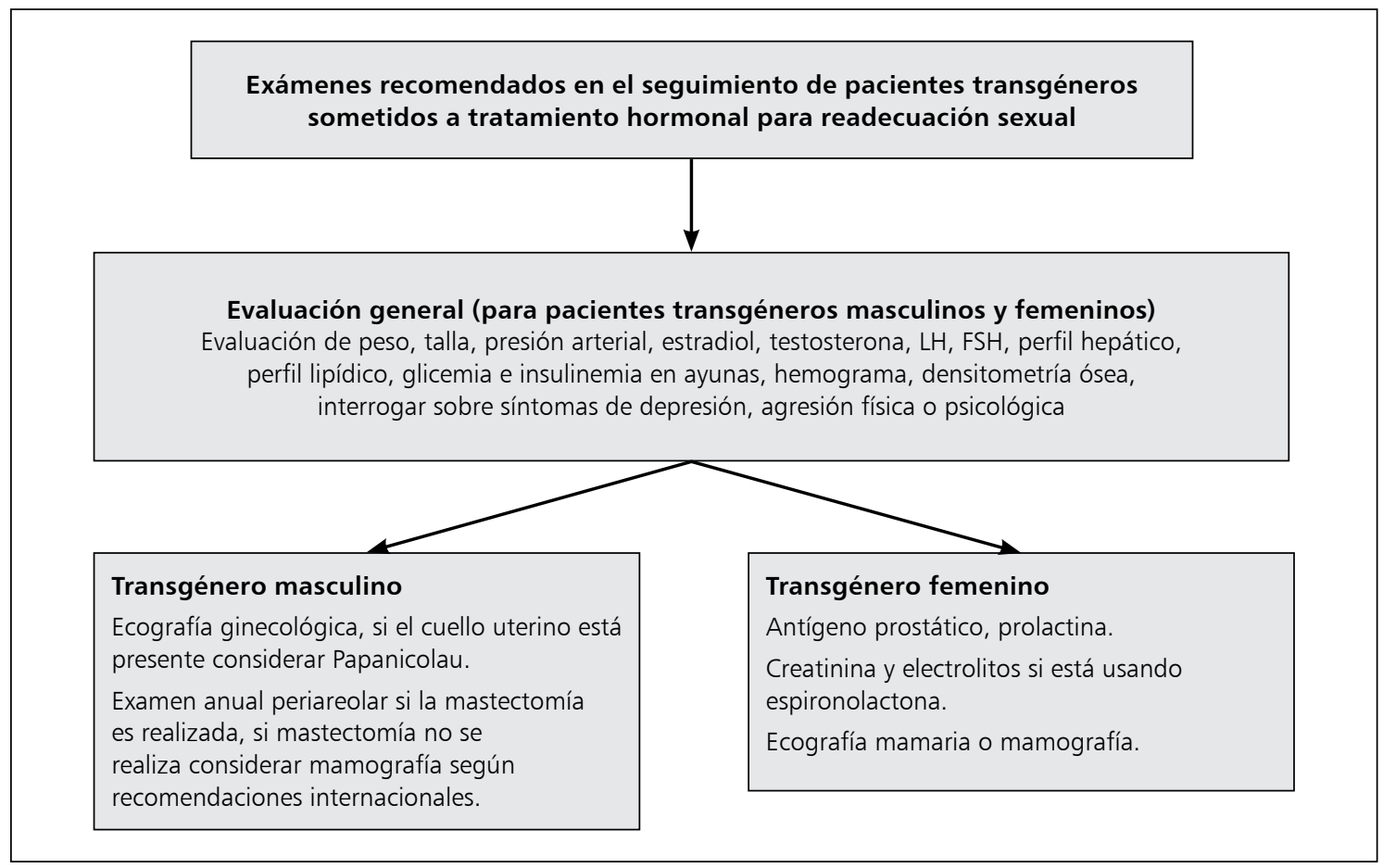

Figura 1.

En adolescentes puberales que tengan evaluación psicológica que confirme que hay discondancia entre el sexo biológico y la identidad de género sentida por ellos y estén en etapa II de Tanner, se sugiere frenar la pubertad con análogos de $\mathrm{GnRH}$, para evitar el desarrollo de mamas y las menstruaciones en los pacientes transgéneros masculinos y el desarrollo de masa muscular masculina en las pacientes transfemeninas. Este tratamiento tiene la ventaja de ser reversible y permitir un período de tiempo (entre los 12 y 16 años) para evaluar la persistencia de necesidad de reasignación corporal sexual y alrededor de los 16 años iniciar la terapia hormonal cruzada ${ }^{26,27,29}$.

La mayoría de los fármacos utilizados en el proceso de hormonación tienen efectos adversos y pueden presentar interacciones con otros medicamentos, que es importante que el médico no especialista tenga en consideración al momento de atender a estos pacientes (Tabla 2). El endocrinólogo realiza controles clínicos y de laboratorio periódicos, pero, en ocasiones las personas trans se automedican, sin controles con especialistas, por lo que el médico debe considerar solicitar exámenes de control en esta población (Figura 1).
El médico debe consultar por síntomas depresivos o ansiosos, así como problemas de violencia o agresión, ya que estos son prevalentes.

\section{Conclusión}

Las personas transgénero constituyen una población que cada vez se hace más visible en los centros de salud de Chile. El/la médico no especialista debe estar preparado para conocer los cuidados en el trato de estas personas, darles acogida y apoyarles si solicitan transitar hacia una readecuación corporal. Es necesario que los/las médicos conozcan que esta población tiene más riesgo de sufrir síntomas depresivos e intentos suicidas, así como agresión física o psicológica, que deben ser buscados en forma dirigida. Además, los/las profesionales deben buscar los probables efectos adversos de medicamentos que usan habitualmente este grupo de pacientes, e indagar posibles prácticas de automedicación, y estar en permanente monitoreo de su estado emocional para brindar apoyo oportuno desde el ámbito de la salud mental. 
Tabla 2. Efectos adversos asociados a la terapia hormonal en el paciente transgénero y recomendaciones de monitorización de exámenes en pacientes en tratamiento de hormonación para readecuación corporal sexual

Transexual femenino
Muy alto riesgo:
Trombosis
Moderado riesgo:
Elevación de enzimas hepáticas
Colelitiasis
Hipertrigliceridemia
Hiperprolactinemia y prolactinoma
Diabetes mellitus tipo 2
Enfermedad coronaria
Enfermedad cerebrovascular
Cáncer de mama

(Adaptado de referencia 34 y 37).

\section{Referencias}

1. Platero R. ¿Qué es transexualidad? En: Platero R. Editor. Transexualidades. Acompañamiento, factores de salud y recursos educativos. Editorial Bellaterra. Barcelona 2014. Pag. 15-37.

2. www.who.int/gender/mainstreaming/ESPwhole.pdf). [Revisado en octubre de 2017].

3. Castillo C, Priego T, Fernández-Tresguerres J. El proceso de diferenciación sexual y biología del transgenerismo. En: Becerra-Fernández A. Editor. Transexualidad, la búsqueda de una identidad. Ediciones Díaz de Santos. Madrid. 2003. Pag 1-20.

4. Asociación de Psicología Americana. Individuos transgéneros e identidad de género. [Revisado en www.iguales. cl/wp-content/uploads/2012/10/IG-APA.pdf, noviembre de 2017].

5. American Psyquiatric Association. Diagnostic and Statistical Manual of Mental Disorders, 5ta Edición. Arlington, VA: American PsyquiatricAssociation Publishing.

6. CIE-11 Clasificación Internacional de Enfermedades. [Revisado en apps.who.int/iris/bitstream/10665/ 42326/ 1/8479034920_spa.pdf, octubre de 2018].

7. www.movilh.cl/trans. [Revisado en octubre de 2017].

8. www.iguales.cl [Revisado en octubre de 2017].

9. Deutsch M. Making it count: Improving estimates of the size of transgender and gender non conforming populations. LGBT Health 2016; 3: 181-5.

10. Olson K. Prepubescent transgender children: what we

\section{Transexual masculino}

Muy alto riesgo:

Policitemia

\section{Moderado riesgo:}

Elevación de enzimas hepáticas

Hipertensión arterial

Dislipidemia

Cáncer uterino

Diabetes mellitus tipo 2

Enfermedad coronaria

Enfermedad cerebrovascular

Cáncer de mama 
with gender identity disorder referred to a pediatric medical center. Pediatric 2012; 129: 418-25.

20. Steensma T, McGuire J, Kreukels B, Beekman A, Cohen-Kettenis $P$. Factors associated with desistence a persistence of childhood gender dysphoria: A quiatitative follow-up study. J Am Acad Child Adolesc Psychiatry 2013; 52: 582-90.

21. Barrientos J, Silva J, Catalán S, Gómez F, Longueira J. Discrimination and Victimization: Parade for Lesbian, Gay, Bisexual and Transgender (LGBT) Pride, in Chile. J Homosex 2010; 57: 6760-75.

22. Barrientos J, Cárdenas M. Construction and Validation of a Subjective Scale of Stigma and Discrimination (SISD) for the Gay Men and Transgender Women Population in Chile (SISD). Sex Res Social Policy 2014; 11 (3): 187-98.

23. Barrientos J, Gómez F, Cárdenas M. Subjetive well-being and levels of clinical symptomatology in a transwomen sample and men who have sex with men in Chile. J Homosex 2016; 63: 11, 1502-16.

24. otdchile.org/biblioteca-otd [Revisado en mayo de 2018].

25. www.who.int/topic/sexual_heath/es/ [Revisado en octubre de 2017].

26. Bockting W, Coleman E, Deutsch M, Guillamon A, Meyer I, Meyer W, et al. Adult development and quality of life of transgender and gender nonconforming people. Curr Opin Endocrinol Diabetes Obes 2016; 23: 188-97.

27. Murad M, Elamin M, García M, Mullan R, Murad A, Erwin $\mathrm{P}$, et al. Hormonal therapy and sex reassignment: a systematic review and meta-analysis of quality of life and psychosocial outcomes. Clinical Endocrinol 2010; 72: 214-31.

28. Hembree W, Cohen-Ketenis P, Gooren L, Hannema S, Meyer W, Murad M, et al. Endocrine treatment of gen- der dysphoric/gender-incongruent persons: an endocrine society clinical practice guideline. J Clin Endocrinol Metab 2017; 102: 3869-903.

29. Tsoi WF. Follow-up study of transsexuals after sexreassignment surgery. Singapore Med J 1993; 34: 515-7.

30. http://www.movilh.cl/documentacion/trans/Circular-Salud-Trans.pdf [Revisado en noviembre de 2017].

31. www.indh.cl/wp-content/uploads/2012/08/Circular-21. pdf [Revisado en noviembre de 2017].

32. web.minsal.cl/portal/url/item/d126e58ba $4 \mathrm{cb}$ 53f5e040010165017912.pdf Vía clínica para la adecuación corporal en personas con incongruencia entre sexo físico e identidad de género. [Revisado en noviembre de 2017].

33. Devoto E, Martínez A, Aravena C. Trastorno de identidad de género Parte I: Definición, epidemiología, etiología, rol del endocrinólogo en diagnóstico y tratamiento. Rev Chil Endocrinol Diabetes 2015; 8: 167-73.

34. Devoto E, Ríos R, Aravena C. Trastorno de identidad de género Parte II: Terapia endocrinológica en el proceso de readecuación corporal. Rev Chil Endocrinol Diabetes 2016; 9: 58-64.

35. www.sstalcahuano.cl/file/diversidad/Resolucion_y_Protocolo_Trans.pdf [Revisado en noviembre de 2017].

36. Pérez Etcheverry M, Delgado Frobel J, Villagrán Varela A, Téllez Téllez R, Martínez García A. Disforia de Género: Caracterización clínica de 28 pacientes. Poster 138 presentado en el XXVIII Congreso de Endocrinología 2017. [Revisado en noviembre de 2017] www.soched. cl/congreso2017/material/poster.html

37. Joseph A, Cliffe C, Hillyard M, Majeed A. Gender identity and the management of the transgender patient: a guide for non-specialists. J R Soc Med 2017; 110: 11452. 